\title{
SOME PROPERTIES OF THE LEVITZKI RADICAL IN ALTERNATIVE RINGS
}

\author{
MICHAEL RICH'
}

(Received 12 June 1980; revised 28 January 1981)

Communicated by R. Lidl

\begin{abstract}
Two local nilpotent properties of an associative or alternative ring $A$ containing an idempotent are shown. First, if $A=A_{11}+A_{10}+A_{01}+A_{00}$ is the Peirce decomposition of $A$ relative to $e$ then if $a$ is associative or semiprime alternative and 3-torsion free then any locally nilpotent ideal $B$ of $A_{i j}$ generates a locally nilpotent ideal $\langle B\rangle$ of $A$. As a consequence $L\left(A_{i j}\right)=A_{i i} \cap L(A)$ for the Levitzi radical $L$. Also bounds are given for the index of nilpotency of any finitely generated subring of $\langle B\rangle$. Second, if $A^{(x)}$ denotes a homotope of $A$ then $L(A) \subseteq L\left(A^{(x)}\right)$ and, in particular, if $A^{(x)}$ is an isotope of $A$ then $L(A)=L\left(A^{(x)}\right)$.
\end{abstract}

1980 Mathematics subject classification (Amer. Math. Soc.): 17 D 05.

\section{Introduction}

It is well known that if an associative or alternative ring $A$ contains an idempotent element $e$ then $A$ can be decomposed into a Peirce decomposition $A=A_{11}+A_{10}+A_{01}+A_{00}$ where $A_{i j}=\{x \in A \mid e x=i x$ and $x e=j x\}$ and that the $A_{i i}$ are subrings of $A$. In [5] Kaplansky has shown that if $b$ is a nilpotent ideal of $A_{i i}$ then the ideal of $A$ generated by $B$ is also nilpotent. From this it is easy to see that if $P$ denotes the prime radical then $P\left(A_{i i}\right)=A_{i i} \cap P(A)$ for $i=0,1$. In the first part of this paper we demonstrate a similar result for local nilpotence. Specifically, if $B$ is a locally nilpotent ideal of $A_{i i}$ then, if $A$ is associative the ideal of $A$ generated by $B$ is locally nilpotent, while if $r$ is semiprime alternative and 3 -torsion free the same result holds. Thus, if $L$

\footnotetext{
${ }^{1}$ This research was partially supported by a Temple University Research Grant. The author also wishes to acknowledge the hospitality of Bar-Ilan University where the research was partly done.

C Copyright Australian Mathematical Society 1982
} 
denotes the Levitzki radical it follows that $L\left(A_{i i}\right)=A_{i i} \cap L(A)$ if $A$ is associative (a result of Anderson [2]) or if $A$ is 3-torsion free alternative.

If $x$ is an element of the alternative ring $A$ then the $x$-homotope $A^{(x)}$ of $A$ is known to be the same additive group as $A$ together with the multiplication $a \cdot{ }_{x} b=(a x) b$. If $x$ is invertible in $A$ then $A^{(x)}$ is called the $x$-isotope of $A$. It is immediate from the characterization of $J(A)$ in [6] that $J(A) \subseteq J\left(A^{(x)}\right)$ for all $x$ in $A$ and if $A^{(x)}$ is an isotope of $A$ then $J(A)=J\left(A^{(x)}\right)$. In the second part of this paper we will prove the analogous result for the Levitzki (locally finite) radical. Specifically, $L(A)$ is a locally nilpotent (finite) ideal of $A^{(x)}$ for all $x$ in $A$, and if $x$ is invertible in $A$ then $L(A)=L\left(A^{(x)}\right)$.

Recall that a non-associative ring $A$ is called alternative if $(x, x, y)=(y, x, x)$ $=0$ for all $x, y \in A$ where the associator $(x, y, z)$ denotes $(x y) z-x(y z)$. A fundamental property of an alternative ring $A$ is that $\left(x_{\sigma(1)}, x_{\sigma(2)}, x_{\sigma(3)}\right)=$ $(\operatorname{sgn} \sigma)\left(x_{1}, x_{2}, x_{3}\right)$ for all $x_{i}$ in $A$ and $\sigma \in S_{3}$. The nucleus, $N(A)$, of $A$ and the center $Z(A)$, of $A$ are defined by $N(A)=\left\{n \in A \mid\left(n, a_{1}, a_{2}\right)=0 \forall a_{1}, a_{2} \in A\right\}$ and $Z(A)=\{z \in N(Z) \mid z a=a z \forall a \in A\}$. The Levitzki (locally finite) radical of the ring (algebra) $A$ is known to be the maximal locally nilpotent (finite) ideal of $A$ where an ideal is called locally nilpotent (finite) if every finitely generated subring (subalgebra) is nilpotent (finite dimensional). It is known that $L(A)$ contains all locally nilpotent (finite) ideals of $A$ and that $L(A / L(A))=0$.

In any alternative ring with an idempotent the following multiplication relations hold among the Peirce subspaces [1], [5].

(1) $A_{i j} A_{j k} \subseteq A_{i k}$ for $i, j, k=0,1$.

(2) $A_{i j} A_{i j} \subseteq A_{j i}$ for $i, j=0,1$.

(3) $A_{i j} A_{k l}=0$ if $j \neq k$ and $(i, j) \neq(k, l)$.

(4) If $x, y, z$ are elements of the Peirce subspaces not all in the same subring $A_{i i}$, then $(x, y, z)=0$ except possibly when two of the elements are in some $A_{i j}$ for $i \neq j$.

Finally, in any alternative ring $A$ the Moufang laws hold for all elements $a, x$, $y$ in $A$.

(5) $(x a x) y=x[a(x y)]$.

(6) $y(x a x)=[(y x) a] x$.

(7) $x(y a) x=(x y)(a x)$.

\section{The Levitzki radical and the Peirce decomposition}

Albert [1] and Kaplansky [5] have noted that if $R$ is an associative or alternative ring and $K$ is an ideal of $R_{i i}$ then the ideal $\langle K\rangle$ of $R$ generated by $K$ is $K+K R_{i j}+R_{j i} K+A_{j i} K A_{i j}$ where $i, j=0,1$. Moreover if $K$ is nilpotent then 
so is $\langle K\rangle$ [5]. In this section we derive a similar result in the case of local nilpotence and show in the course of the proof how to find an upper bound to the index of nilpotency of any finitely generated subring of $\langle K\rangle$. Henceforth we shall assume that $A$ is an alternative ring.

Lemma 1. Let $K$ be a locally nilpotent (finite) ideal of $A_{i i}$ such that $\langle K\rangle=K+$ $K A_{i j}+A_{j i} K+A_{j i} K A_{i j} \subseteq N(A)$. Then $A_{j i} K A_{i j}$ is a locally nilpotent (finite) ideal of $A$.

Proof. For convenience we discuss the case of $i=1$. The case $i=0$ is obtained by interchanging 0 and 1 throughout. It is immediate from (4) that $A_{01} K A_{10}$ is an ideal of $A_{00}$. To see that it is locally nilpotent when $K$ is, consider an arbitrary finite subset $B$ of $A_{01} K A_{10}$. It suffices to replace $B$ by the finite set $B^{\prime}$ of terms $x_{01} k x_{10}$ appearing in the various elements of $B$ since the span of $B^{\prime}$ contains $B$. Thus, we may as well assume that

$$
B=\left\{x_{\delta 1}^{(1)} k_{1} x_{10}^{(1)}, x_{01}^{(2)} k_{2} x_{10}^{(2)}, \ldots, x_{01}^{(r)} k x_{10}^{(r)}\right\}
$$

for some $k_{s} \in K, x_{s t}^{(m)} \in A_{s t}$. Consider the finite subset $T=\left\{k_{i} x_{10}^{(i)} x_{01}^{())} \mid i, j=\right.$ $1,2, \ldots, r\}$ of $K$. Since $K$ is locally nilpotent, $T$ generates a nilpotent subring, say of index $n-1$. Then the subring generated by $B$ is nilpotent of index at most $n$. For, since $K A_{10} \subseteq\langle K\rangle \subseteq N(A)$ we have, by repeated application of (1) and (4),

$$
\left(x_{\delta 1}^{(i)} k_{i} x_{10}^{(i)}\right)\left(x_{\delta 1}^{(i)} k_{j} x_{10}^{(i)}\right)=x_{\delta}^{(i)}\left[k_{i}\left(x_{10}^{(i)} x_{\delta 1}^{(i)}\right) k_{j}\right] x_{i \beta}^{(j)} .
$$

Therefore any product

$$
\left(x_{\delta 1}^{\left(i_{1}\right)} k_{i_{1}} x_{10}^{\left(i_{1}\right)}\right)\left(x_{\delta 1}^{\left(i_{1}\right)} k_{i_{2}} x_{10}^{\left(i_{2}\right)}\right) \cdots\left(x_{\delta 1}^{\left(i_{1}\right)} k_{i_{n}} x_{10}^{\left(i_{1}\right)}\right)
$$

of $n$ elements of $B$ reduces to

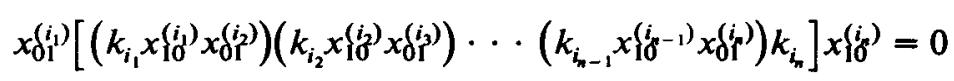

since it contains a string of $n-1$ elements of $T$. Therefore $A_{01} K A_{10}$ is a locally nilpotent ideal of $A_{00}$. For the case in which $K$ is given to be a locally finite ideal of an algebra $A$ note that the subalgebra generated by $T$ is finite dimensional with basis $l_{1}, l_{2}, \ldots, l_{t}$ say. Then the argument just given shows that the subalgebra generated by $B$ is spanned by the elements $x_{01}^{(i)} l_{j} x_{10}^{(k)}$ for some $i$, $k=1, \ldots, n$ and $j=1,2, \ldots, t$. Therefore it is finite dimensional and $A_{01} K A_{10}$ is locally finite.

LEMMA 2. If $A$ is $n$-torsion free and $L$ is the Levitzki radical then $\bar{A}=A / L(A)$ is also n-torsion free. 
Proof. Suppose that $n \bar{a}=\overline{0}$ for some $a \in A$. Then $n a \in L(A)$. We show that the ideal $(L, a)$ generated by $L(A)$ and $a$ is locally nilpotent, from which it follows that $a \in L(A)$. Note that a typical element of $(L, a)$ is of the form $l+m(a)$ for some $l \in L(A)$ and $m \in M\left(A^{\prime}\right)$ where $A^{\prime}$ is the ring obtained after adjoining an identity element to $A$ and $M\left(A^{\prime}\right)$ is the multiplication ring of $A^{\prime}$. Now if we pick any finite subset $T=\left\{t_{1}, t_{2}, \ldots, t_{s}\right\}$ of $(L, a)$ then, since $n a \in L(A)$, the subring generated by $n T=\left\{n t_{1}, n t_{2}, \ldots, n t_{s}\right\}$ is nilpotent, say of index $k$. Thus, if we consider any product $t=t_{i_{1}}, t_{i_{2}}, \ldots, t_{i_{k}}$ of elements $t_{i,} \in T$ (regardless of the association) it follows that $n^{k_{t}}=0$. But since $A$ is $n$-torsion free this means that $t=t_{i_{1}} t_{i_{2}}, \ldots, t_{i_{k}}=0$. Hence, the subring generated by $T$ is nilpotent of index $k$ and $(L, a)$ is locally nilpotent. Thus, $(L, a) \subseteq$ $L(A)$ so that $a \in L(A)$ and $\bar{a}=0$. We have shown that $\bar{A}$ is $n$-torsion free.

In the following $L_{i}$ will denote $L\left(A_{i i}\right)$ for $i=0,1$.

THEOREM 1. If $A$ is a semiprime 3-torsion free alternative ring with an idempotent element $e$ and $K$ is a locally nilpotent ideal of $A_{i i}$ then $\langle K\rangle$ is a locally nilpotent ideal of $A$.

Proof. We shall prove the theorem for the case $i=1$. The case $i=0$ is obtained by interchanging the indices 0 and 1 throughout the proof. Since $A$ is semiprime and 3-torsior. free we may apply Corollary 7.7 of [9] to obtain that either

(i) $\langle K\rangle \cap Z(A) \neq 0$ or

(ii) $\langle K\rangle \subseteq N(A)$.

If (i) holds then there is a non-zero element $k=k_{1}+k_{10}+k_{01}+k_{0} \in\langle K\rangle \cap$ $Z(A)$. Since $e k=k e$ it follows that $k_{10}=k_{01}=0$ so that $k=k_{1}+k_{0}$. But then $k_{1} \in Z\left(A_{11}\right)$ and since $k_{1}$ is nilpotent, $k_{1} A_{11}$ is a non-zero nilpotent ideal of $A_{11}$. But then by [5] $\left\langle k_{1} A_{11}\right\rangle$, the ideal of $A$ generated by $k_{1} A_{11}$, is a non-zero nilpotent ideal of $A$ contradicting the fact that $A$ is semiprime.

Assume then that $\langle K\rangle=K+K R_{10}+R_{01} K+R_{01} K R_{10} \subseteq N(A)$. Then by (1) and (3) $\left(K R_{10}\right)^{2}=\left(K R_{10} K\right) R_{10}=0$. Similarly $\left(R_{01} K\right)^{2}=0$. Consider an arbitrary finite set $S$ of elements of the Peirce spaces of $\langle K\rangle$. Then

$$
\begin{aligned}
S=\{ & k_{1}^{(1)}, k_{1}^{(2)}, \ldots, k_{1}^{(p)}, k_{0}^{(1)}, k_{0}^{(2)}, \ldots, k_{0}^{(q)}, k_{1}^{(p+1)} r_{10}^{(1)}, \ldots, \\
& \left.k^{(p+m)} r_{10}^{(m)}, r_{01}^{(1)} k^{(p+m+1)}, r_{01}^{(2)} k^{(p+m+2)}, \ldots, r_{01}^{(s)} l_{1}^{(p+m+s)}\right\}
\end{aligned}
$$

with $k_{1}^{(d)} \in K, r_{i j}^{(d)} \in A_{i j}$, and $k_{0}^{(d)} \in R_{01} K R_{10}$. Thus by Lemma $1 k_{0}^{(d)} \in L_{0}$ for all $d$. 
We wish to show that the subring [S] of $A$ generated by $S$ is nilpotent. To see this first look at the set

$$
E=\left\{k_{1}^{(1)}, k_{1}^{(2)}, \ldots, k^{(p+m+s)}, k_{\delta}^{(1)}, k_{\delta}^{(2)}, \ldots, k_{\delta}^{(q)}\right\}
$$

of elements of $K \cup L_{0}$. Since $K L_{0}=L_{0} K=0, K+L_{0}$ is locally nilpotent. Therefore, the subring $[E]$ generated by $E$ is nilpotent, say of index $n$. Next consider the set $C$ where

$$
\begin{aligned}
& C=E \cup\left\{r_{81}^{(i)} k^{\left(i_{1}\right)} k_{1}^{\left(i_{2}\right)} \cdots k_{1}^{\left(i_{w}\right)} r_{i \sigma \mid}^{(i)}\right. \\
& \left.1<j \leqslant s, 1 \leqslant i \leqslant m, 1 \leqslant i_{1} \leqslant p+m+s, 0<w<n-1\right\} \\
& \cup\left\{r_{10}^{(i)} k_{0}^{\left(i_{1}\right)} k_{0}^{\left(i_{2}\right)} \cdots k_{0}^{\left(i_{w}\right)} r_{\delta}^{(i)} \mid\right. \\
& \left.1 \leqslant j \leqslant s, 1 \leqslant i<m, 1 \leqslant i_{l}<p+m+s, 0<w<n-1\right\} \\
& \cup\left\{k_{\mathrm{l}}^{(h)} r_{10}^{(i)} r_{01}^{(j)} \mid 1<h<p+m+s, 1<i<m, 1<j<s\right\} \\
& \cup\left\{k_{0}^{(g)} r_{0}^{(j)} r_{10}^{(i)} \mid 1<g<n-1,1<j<s, 1<i<m\right\} .
\end{aligned}
$$

In view of Lemma $1, C$ is a subset of $K+L_{0}$. Thus, $[C]$, the subring generated by $C$ is nilpotent of index $t$ for some $t>n$.

We now claim that $[S]$ is nilpotent of index $<2 n t$. For suppose that $u$ was a non-zero monomial of $[S]$ of degree $2 n t$ in the generators of $[S]$. Then since $S \subseteq N(A), u$ must take one of the forms:

(i) $u=k_{1}^{()} M$,

(ii) $u=s_{10} M$,

(iii) $u=k_{0}^{(j)} M$,

(iv) $u=s_{01} M$

for some $s_{i j} \in S_{i j}=S \cap A_{i j}$ and $M \in[S]$. Suppose that $u$ is of the form (i). Then, by (3), since $s_{i j}^{2}=0$ for $i \neq j, u$ can be decomposed into a product of: First a product of elements of $S_{11}$, then an element of $S_{10}$, then a product of a number (possibly none) of elements of $S_{00}$, followed by an element of $S_{01}$, etc. Since $[E]$ is nilpotent of index $n$ there is no sequence of $n$ consecutive elements of $E$ in $u$. Thus,

$$
u \in S_{11}^{d_{1}} S_{10} S_{00}^{d_{2}} S_{01} S_{11}^{d_{3}} S_{10} \ldots
$$

for $1 \leqslant d_{1} \leqslant n-1,0<d_{i} \leqslant n-1$ for $i>1$. Since $u$ is of degree $2 n t$ in $S$ and $d_{i} \leqslant n-1$ we are guaranteed that $u$ begins with at least $2 t$ blocks of the form $s_{i i}^{d} S_{i j}$, that is,

$$
u \in S_{11}^{d_{1}} S_{10} S_{03}^{d_{2}} S_{01} S_{11}^{d_{3}} \cdots S_{10} S_{00^{2}}^{d_{2}} S_{01} N
$$

with $N \in[S]$. Thus,

$$
u \in S_{11}^{d_{1}}\left(S_{10} S_{00}^{d_{2}} S_{01}\right) S_{11}^{d_{3}}\left(S_{10} S_{00}^{d_{1}} S_{01}\right) \cdots\left(S_{10} S_{00}^{d_{t}} S_{01}\right) N .
$$


If $d_{2 i}>1$ for each $i$ then $u$ contains at least $t$ consecutive factors from $C$. Thus, $u=0-\mathrm{a}$ contradiction. Assume then that $d_{2 i}=0$ for some $i$. Then $S_{10} S_{00}^{d_{2}} S_{01}=$ $S_{10} S_{01}$. Now a term of $S_{10} S_{01}$ is of the form $k_{1}^{(h)} r_{10}^{(i)} r_{01}^{())} k_{1}^{(l)}$. Since the elements $k_{1}^{()}$ and $k_{1}^{(}{ }^{\prime}{ }_{10}^{(} r_{01}^{()}$are all elements of $C$, it is clear that elements of $S_{10} S_{01}$ are also generated by element of $C$. Therefore, here also $u$ contains at least $t$ consecutive factors of $C$. Thus, $u=0$.

Suppose then that $u$ is of the form (ii). Then $u \in S_{10} S_{00}^{d_{1}} S_{01} S_{1\}}^{d_{1}} S_{10} S_{00}^{d_{3}} S_{01} \ldots$ for $0<d_{i}<n-1$. Since $u$ is of degree $2 n t$ in $S$ it follows that

$$
u \in S_{10} S_{00}^{d_{1}} S_{01} S_{11}^{d_{2}} \cdots S_{00^{t-1}}^{d_{2}} S_{01} S_{11}^{d_{2 t} N}
$$

for some $N \in[S]$. Thus,

$$
u \in\left(S_{10} S_{00}^{d_{1}} S_{01}\right) S_{11}^{d_{2}}\left(S_{10} S_{00}^{d_{3}} S_{01}\right) \cdots\left(S_{10} S_{00}^{d_{2 t-1}} S_{01}\right) S_{11^{2}}^{d^{2}} N \text {. }
$$

Then, as in case (i) $u$ must contain at least $t$ consecutive factors from $C$. Thus, $u=0$. The cases (iii) and (iv) are treated similarly. Thus, any monomial of $[S]$ of degree $2 n t$ reduces to zero so that $[S]$ is nilpotent of index $<2 n t$. We have thus shown that if $K \neq 0$ then $\langle K\rangle$ is a locally nilpotent ideal of $A$.

Since the whole point of our conditions that $A$ be semiprime and 3-torsion free is to insure that $\langle K\rangle \subseteq N(A)$ we have immediately:

COROLlaRY 1. If $R$ is an associative ring with idempotent $e$ and $K$ is a locally nilpotent ideal of $R_{i i}$ then $\langle K\rangle$ is a locally nilpotent ideal of $R$.

COROLlaRY 2. If $A$ is a 3-torsion free alternative ring with an idempotent element and $L$ is its Levitzki radical, then $L\left(A_{i i}\right)=A_{i i} \cap L(A)$.

Proof. To begin, note that $A_{i i} \cap L(A)$ is a locally nilpotent ideal of $A_{i i}$. Therefore $A_{i i} \cap L(A) \subseteq L\left(A_{i i}\right)$. For the reverse inclusion, assume first that $A$ is Levitzki semisimple. Now if $A_{i i}$ is not Levitzki semisimple, $L_{i} \neq 0$. But then, by Theorem $1,\left\langle L_{i}\right\rangle$ is a locally nilpotent ideal $A$ for a contradiction. Therefore, $A$ Levitzki semisimple implies that $A_{i i}$ is Levitzki semisimple.

In the general case when $L(A) \neq 0$ let $\bar{A}=A / L(A)$ so that $\bar{A}$ is Levitzki semisimple. Also $\bar{A}$ is 3-torsion free by Lemma 2 . Therefore $L\left((\bar{A})_{i i}\right)=0$. Now it is an easy exercise to see that $(\bar{A})_{i i} \approx A_{i i} /\left(A_{i i} \cap L(A)\right)$. Therefore, $L\left(A_{i i} / A_{i i} \cap L(A)\right)=0$ and $L\left(A_{i i}\right) \subseteq A_{i i} \cap L(A)$ to complete the proof.

Finally, we note that Corollary 1 provides a direct proof to the result of Anderson [2] that for associative rings $R, L\left(R_{i i}\right)=R_{i i} \cap L(R)$. 


\section{The Levitzki radical and homotopy}

It is known that if $A^{(x)}$ is an isotope of $A$ then $J\left(A^{(x)}\right)=J(A)$ [6] and $P\left(A^{(x)}\right)=P(A)$ [3]. In this section we derive the same result for the Levitzki radical by first analyzing the more general case of homotopy.

In the following, $R_{b}$ denotes the usual right multiplication operator, that is, $a R_{b}=a b$. Similarly $a R_{b}^{(x)}=(a x) b$ for all $a, b \in A$.

LEMMA 3. If $a, i_{1}, i_{2}, \ldots, i_{n}, x$ are arbitrary elements of $A$, then

$$
a R_{i_{1}}^{(x)} R_{i_{2}}^{(x)} \cdots R_{i_{n}}^{(x)}=\left\{\begin{array}{cc}
a R_{x i_{1} x} R_{i_{2}} R_{x i_{3} x} \cdots R_{x i_{n-1} x} R_{i_{n}} & \text { if } n \text { is even, } \\
(a x) R_{i_{1}} R_{x i_{2} x} R_{i_{3}} \cdots R_{x i_{n-1} x} R_{i_{n}} & \text { if } n \text { is odd. }
\end{array}\right.
$$

Proof. The proof is by induction on $n$. The case $n=1$ is obvious form the definition of $R_{i}^{(x)}$. In the case $n=2$ we have $a R_{i_{1}}^{(x)} R_{i_{2}}^{(x)}=\left(\left[(a x) i_{1}\right] x\right) i_{2}=$ [ $\left.a\left(x i_{1} x\right)\right] i_{2}$ by (6). Therefore $a R_{i_{1}}^{(x)} R_{i_{2}}^{(x)}=a R_{x i_{1} x} R_{i_{2}}$. Assume then that the result is true for $k<n$ and let $u=a R_{i_{1}}^{(x)} R_{i_{2}}^{(x)} \cdots R_{i_{n}}^{(x)}$. If $w=a R_{i_{1}}^{(x)} \boldsymbol{R}_{i_{2}}^{(x)} \cdots R_{i_{n-2}}^{(x)}$ then $u=w R_{i_{n-1}}^{(x)} R_{i_{n}}^{(x)}=\left(\left[(w x) i_{n-1}\right] x\right) i_{n}=\left[w\left(x i_{n-1} x\right)\right] i_{n}$. By the induction hypothesis $w$ has the desired form. Since $n$ and $n-2$ have the same parity it follows that $u$ has the desired form. Thus, the lemma is true.

LEMMA 4. If $a, i_{1}, i_{2}, \ldots, i_{n}, x$ are arbitrary elements of $A$ and $u=$ $a R_{i_{1}}^{(x)} R_{i_{2}}^{(x)} \cdots R_{i_{n}}^{(x)}$ then $x u x=(x a x) R_{i_{1} x} \cdots R_{i_{n} x}$

Proof. The result again follows by induction on $n$. In case $n=1, x u x=$ $x\left[(a x) i_{1}\right] x=(x a x)\left(i_{1} x\right)$ by (7). Assume that the result holds for $n-1$ and $u=a R_{i_{1}}^{(x)} R_{i_{2}}^{(x)} \cdots R_{i_{n}}^{(x)}$. Let $w=a R_{i_{1}}^{(x)} \cdots R_{i_{n-1}}^{(x)}$. Then $x u x=x\left(w R_{i_{n}}^{(x)}\right) x$ $=x\left((w x) i_{n}\right) x=(x w x)\left(i_{n} x\right)$. By the induction hypothesis $x w x$ $=(x a x) R_{i_{1} x} \cdots R_{i_{n-1} x}$. Therefore $x u x=(x w x)\left(i_{n} x\right)=(x a x) R_{i_{1} x} \cdots R_{i_{n-1} x} R_{i_{n} x}$ to complete the proof.

LEMMA 5. If $a, i_{1}, i_{2}, \ldots, i_{n}, x$ are arbitrary elements of $A$ and $u=$ $a^{(x)} R_{i_{1}} R_{i_{2}}^{(x)} \cdots R_{i_{n}}^{(x)}$ then

$$
u x=\left\{\begin{array}{cc}
(a x) R_{i_{1}} R_{x i_{2} x} R_{i_{3}} \cdots R_{i_{n-1}} R_{x i_{n} x} & \text { if } n \text { is even, } \\
a R_{x i i_{1} x} R_{i_{2}} R_{x i_{3} x} \cdots R_{i_{n-1}} R_{x i_{n} x} & \text { if } n \text { is odd. }
\end{array}\right.
$$


Proof. We once again proceed by induction on $n$. If $n=1$ then $u x=$ $\left(a R_{i_{1}}^{(x)}\right) x=\left((a x) i_{1}\right) x=a\left(x i_{1} x\right)=a R_{x i_{1} x}$ by (6). Similarly if $n=2$ then $u x=$ $\left[a R_{i_{1}}^{(x)} R_{i_{2}}^{(x)}\right] x=\left[\left[\left[(a x) i_{1}\right] x\right] i_{2}\right] x=\left[(a x) i_{1}\right]\left(x i_{2} x\right)$ by (6) again. Therefore $u x=$ (ax) $R_{i} R_{x i, x}$ as required. Assume that the rsult holds for $k<n$ and that $u=$ $R_{i_{1}}^{(x)} R_{i_{2}}^{(x)} \cdots R_{i_{n}}^{(x)}$. As in Lemma 3 let $w=a R_{i_{1}}^{(x)} R_{i_{2}}^{(x)} \cdots R_{i_{n-2}}^{(x)}$. Then

$$
\begin{aligned}
u x & =\left(w R_{i_{n-1}}^{(x)} R_{i_{n}}^{(x)}\right) x=\left[\left[\left[(w x) i_{n-1}\right] x\right] i_{n}\right] x \\
& =\left[(w x) i_{n-1}\right]\left(x i_{n} x\right)=(w x) R_{i_{n-1}} R_{x i_{n} x} .
\end{aligned}
$$

Now, by the induction hypothesis $w x$ has the desired form. Since $n$ and $n-2$ have the same parity it follows that $u x$ has the desired form. Thus, the lemma holds by mathematical induction.

In the following we shall denote by $e$ the identity element of $B^{(x)}$ if it has one, and, if not, $e$ will denote the identity element of $\left(B^{(x)}\right)^{\prime}$ where $\left(B^{(x)}\right)^{\prime}$ is the ring obtained after imbedding $B^{(x)}$ into a ring with identity in the usual way.

THEOREM 2. If $x$ is an arbitrary element of the alternative ring $A$ and $I$ is $a$ locally nilpotent ideal of $A$, then $I^{(x)}$ is a locally nilpotent ideal of the homotope $A^{(x)}$

Proof. Let $B^{(x)}$ be a finitely generated subring of $I^{(x)}$ generated by the elements $i_{1}, i_{2}, \ldots, i_{n}$. Then, since $I$ is a locally nilpotent ideal the set $D=\left\{i_{1}\right.$, $\left.i_{2}, \ldots, i_{n}, i_{1} x, i_{2} x, \ldots, i_{n} x, x i_{1} x, x i_{2} x, \ldots, x i_{n} x\right\}$ generates a nilpotent subring $[D]$ of $A$. Assume that $[D]^{k}=0$. Now, by [7, Proposition 3] every element $b$ of $B^{(x)}$ is a linear combination of second degree monomials, so that without loss of generality we may in the course of the proof assume that each element $b$ of $B^{(x)}$ is a second degree monomial in $i_{1}, i_{2}, \ldots, i_{n}$ (that is, $b=e R_{c_{1}}^{(x)} R_{c_{2}}^{(x)} \cdots R_{c_{m}}^{(x)}$ ), where $c_{t}=e R_{i_{(1)}}^{(x)} R_{i_{(2)}}^{(x)} \cdots R_{i_{(s)}}^{(x)}$ for some positive integers $m, s$, and $t(s)$ and where $1 \leqslant t(j) \leqslant n$ for all $j=1,2, \ldots, s$. Since $b=c_{1} R_{c_{2}}^{(x)} \cdots R_{c_{m}}^{(x)}$, by Lemma 3 either

(i) $b=c_{1} R_{x c_{2} x} R_{c_{3}} \cdots R_{x c_{m-1} x} R_{c_{m}}$ or

(ii) $b=\left(c_{1} x\right) R_{c_{2}} R_{x c_{3} x} R_{c_{4}} \cdots R_{x c_{m-1} x} R_{x c_{m-1} x} R_{c_{m}}$ depending on the parity of $m$. Similarly, since $c t=i_{t(1)} R_{i(2)}^{(x)} \cdots R_{i_{i(s)}^{(x)}}^{(x)}$ we have that either

$$
c_{t}=i_{t(1)} R_{x i_{(2)} x} R_{i_{t(3)}} \cdots R_{x i_{(s-1)} x} R_{i_{(x, s)}}
$$

or

$$
c_{t}=\left(i_{t(1)} x\right) R_{i_{(2)}} R_{\left.x i_{(3)}\right)^{x}} \cdots R_{x i_{(s,-1) x}} R_{i_{t_{(s)}}} .
$$

In either case, $c_{t}$ is a monomial of $I$ of degree $s$ in the generators from $D$.

Since $c_{t}=i_{t(1)} R_{i_{(2)}}^{(x)} \cdots R_{i_{(s)}}^{(x)}$ it follows from Lemma 4 that

$$
x c_{t} x=\left(x i_{t(1)} x\right) R_{i_{(2(2)} x} R_{i_{(3,3)} x} \cdots R_{i_{(1,3)} x} .
$$


Therefore $x c_{t} x$ is a monomial of $I$ of degree $s$ in the generators $x i_{t_{1}}$, $i_{t_{2}} x, \ldots, i_{t(s)} x$ of $D$. Similarly, by Lemma $5, c_{t} x$ is a monomial of $I$ of degree $s$ in generators of $D$. Thus, in both cases (i) and (ii) if $b$ is of degree $u$ in the generators $\left\{i_{1}, i_{2}, \ldots, i_{n}\right\}$ as an element of $I^{(x)}$ then $b$ is of degree $u$ in the generators $D=\left\{i_{1}, i_{2}, \ldots, i_{n}, i_{1} x, \ldots, i_{n} x, x i_{1} x, \ldots, x i_{n} x\right\}$ as an element of $I$. Since $[D]^{k}=0$ it follows that any element of $B^{(x)}$ of degree $k$ in $i_{1}, i_{2}, \ldots, i_{n}$ reduces to zero. Thus, $B^{(x)}$ is nilpotent of degree $<k$ and $I^{(x)}$ is locally nilpotent.

Since $A$ is a homotope of $A^{(x)}$ when $x$ is an invertible element of $A$ we have:

COROllary. If $A^{(x)}$ is an isotope of $A$ then $L\left(A^{(x)}\right)=L(A)$.

Note. The crux of the proof of the theorem is that $B^{(x)} \subseteq[D]$. Thus, if $A$ is an algebra over a field $F$ and $[D]$ is finite dimensional then it follows that $B^{(x)}$ is finite dimensional. Therefore, if $I$ is locally finite we conclude in the same way that $I^{(x)}$ is locally finite.

\section{References}

[1] A. A. Albert, (1952), 'On simple alternative rings', Canad. J. Math. 4, 129-135.

[2] T. Anderson, (1974), 'A note on strong radicals', Acta Math. Acad. Sci. Hungar. 25, 5-6.

[3] T. S. Erickson, 'The Jordan prime radical in alternative algebras', unpublished.

[4] I. N. Herstein, (1968), 'Noncommutative rings' (The Math. Assoc. of Amer.).

[5] I. Kaplansky, (1951), 'Semi-simple alternative rings', Portugal. Math. 10, 37-50.

[6] K. McCrimmon, (1971), 'A characterization of the Jacobson-Smiley Radical', J. Algebra 18, $565-573$.

[7] K. McCrimmon, (1973), 'Alternative algebras satisfying polynomial identities', J. Algebra 24, 283-292.

[8] M. Rich, (1980), 'Nilpotent ideals in alternative rings', Bull. Canad. Math. Soc. 23, 299-303.

[9] M. Slater, (1968), 'Ideals in semiprime alternative rings', J. Algebra 8, 60-76.

Department of Mathematics

Temple University

Philadelphia, Pennsylvania 19122

U.S.A. 